\title{
Observations of selected AGN with HESS
}

F. Aharonian ${ }^{1}$, A. G. Akhperjanian ${ }^{2}$, A. R. Bazer-Bachi ${ }^{3}$, M. Beilicke ${ }^{4}$, W. Benbow ${ }^{1}$, D. Berge ${ }^{1}$, K. Bernlöhr ${ }^{1,5}$, C. Boisson ${ }^{6}$, O. Bolz ${ }^{1}$, V. Borrel ${ }^{3}$, I. Braun ${ }^{1}$, F. Breitling ${ }^{5}$, A. M. Brown ${ }^{7}$, P. M. Chadwick ${ }^{7}$, L.-M. Chounet ${ }^{8}$, R. Cornils ${ }^{4}$, L. Costamante ${ }^{1,20}$, B. Degrange ${ }^{8}$, H. J. Dickinson ${ }^{7}$, A. Djannati-Ataï ${ }^{9}$, L. O'C. Drury ${ }^{10}$, G. Dubus ${ }^{8}$, D. Emmanoulopoulos ${ }^{11}$, P. Espigat ${ }^{\text {}}$, F. Feinstein ${ }^{12}$, G. Fontaine ${ }^{8}$, Y. Fuchs ${ }^{13}$, S. Funk ${ }^{1}$, Y. A. Gallant ${ }^{12}$, B. Giebels ${ }^{8}$, S. Gillessen ${ }^{1}$, J. F. Glicenstein ${ }^{14}$, P. Goret ${ }^{14}$, C. Hadjichristidis ${ }^{7}$, M. Hauser ${ }^{11}$, G. Heinzelmann ${ }^{4}$, G. Henri ${ }^{13}$, G. Hermann ${ }^{1}$, J. A. Hinton ${ }^{1}$, W. Hofmann ${ }^{1}$, M. Holleran ${ }^{15}$, D. Horns ${ }^{1}$, A. Jacholkowska ${ }^{12}$, O. C. de Jager ${ }^{15}$, B. Khélifi ${ }^{1}$, Nu. Komin ${ }^{5}$, A. Konopelko ${ }^{1,5}$, I. J. Latham ${ }^{7}$, R. Le Gallou ${ }^{7}$, A. Lemière ${ }^{9}$, M. Lemoine-Goumard ${ }^{8}$, N. Leroy ${ }^{8}$, T. Lohse ${ }^{5}$, J. M. Martin ${ }^{6}$, O. Martineau-Huynh ${ }^{16}$, A. Marcowith ${ }^{3}$, C. Masterson ${ }^{1,20}$, T. J. L. McComb ${ }^{7}$, M. de Naurois ${ }^{16}$, S. J. Nolan ${ }^{7}$, A. Noutsos ${ }^{7}$, K. J. Orford ${ }^{7}$, J. L. Osborne ${ }^{7}$, M. Ouchrif ${ }^{16,20}$, M. Panter ${ }^{1}$, G. Pelletier ${ }^{13}$, S. Pita ${ }^{9}$, G. Pühlhofer ${ }^{1,11}$, M. Punch ${ }^{9}$, B. C. Raubenheimer ${ }^{15}$, M. Raue ${ }^{4}$, J. Raux ${ }^{16}$, S. M. Rayner ${ }^{7}$, A. Reimer ${ }^{17}$, O. Reimer ${ }^{17}$, J. Ripken ${ }^{4}$, L. Rob ${ }^{18}$, L. Rolland ${ }^{16}$, G. Rowell ${ }^{1}$, V. Sahakian ${ }^{2}$, L. Saugé ${ }^{13}$, S. Schlenker $^{5}$, R. Schlickeiser ${ }^{17}$, C. Schuster ${ }^{17}$, U. Schwanke ${ }^{5}$, M. Siewert ${ }^{17}$, H. Sol ${ }^{6}$, D. Spangler ${ }^{7}$, R. Steenkamp ${ }^{19}$, C. Stegmann ${ }^{5}$, J.-P. Tavernet ${ }^{16}$, R. Terrier ${ }^{9}$, C. G. Théoret ${ }^{9}$, M. Tluczykont ${ }^{8,20}$, G. Vasileiadis ${ }^{12}$, C. Venter ${ }^{15}$, P. Vincent ${ }^{16}$, H. J. Völk ${ }^{1}$, and S. J. Wagner ${ }^{11}$

\footnotetext{
${ }^{1}$ Max-Planck-Institut für Kernphysik, Heidelberg, Germany; e-mail: wystan. benbow@mpi-hd.mpg.de; ${ }^{2}$ Yerevan Physics Institute, Armenia; ${ }^{3}$ Centre d'Étude Spatiale des Rayonnements, CNRS/UPS, Toulouse, France; ${ }^{4}$ Universität Hamburg, Institut für Experimentalphysik, Germany; ${ }^{5}$ Institut für Physik, Humboldt-Universität zu Berlin, Germany; ${ }^{6}$ LUTH, UMR 8102 du CNRS, Observatoire de Paris, Section de Meudon, France; ${ }^{7}$ University of Durham, Department of Physics, UK; ${ }^{8}$ Laboratoire Leprince-Ringuet, IN2P3/CNRS, École Polytechnique, Palaiseau, France; ${ }^{9}$ APC, Paris, France ${ }^{\star}$; ${ }^{10}$ Dublin Institute for Advanced Studies, Ireland; ${ }^{11}$ Landessternwarte, Königstuhl, Heidelberg, Germany; ${ }^{12}$ Laboratoire de Physique Théorique et Astroparticules, IN2P3/CNRS, Université Montpellier II, France; ${ }^{13}$ Laboratoire d'Astrophysique de Grenoble, INSU/CNRS, Université Joseph Fourier, France; ${ }^{14}$ DAPNIA/DSM/CEA, CE Saclay, Gif-sur-Yvette, France; ${ }^{15}$ Unit for Space Physics, North-West University, Potchefstroom, South Africa; ${ }^{16}$ Laboratoire de Physique Nucléaire et de Hautes Energies, IN2P3/CNRS, Universités Paris VI \& VII, France; ${ }^{17}$ Institut für Theoretische Physik, Lehrstuhl IV, Ruhr-Universität Bochum, Germany; ${ }^{18}$ Institute of Particle and Nuclear Physics, Charles University, Prague, Czech Republic; ${ }^{19}$ University of Namibia, Windhoek, Namibia; ${ }^{20}$ European Associated Laboratory for Gamma-Ray Astronomy, jointly supported by CNRS and MPG
}

Received 19 May 2005 / Accepted 16 June 2005

\begin{abstract}
A sample of selected active galactic nuclei (AGN) was observed in 2003 and 2004 with the High Energy Stereoscopic System (HESS), an array of imaging atmospheric-Cherenkov telescopes in Namibia. The redshifts of these candidate very-highenergy (VHE, $>100 \mathrm{GeV}) \gamma$-ray emitters range from $z=0.00183$ to $z=0.333$. Significant detections were already reported for some of these objects, such as PKS 2155-304 and Markarian 421. Marginal evidence $(3.1 \sigma)$ for a signal is found from large-zenith-angle observations of Markarian 501, corresponding to an integral flux of $I(>1.65 \mathrm{TeV})=\left(1.5 \pm 0.6_{\text {stat }} \pm 0.3_{\text {syst }}\right) \times$ $10^{-12} \mathrm{~cm}^{-2} \mathrm{~s}^{-1}$ or $\sim 15 \%$ of the Crab Nebula flux. Integral flux upper limits for 19 other AGN, based on exposures of $\sim 1$ to $\sim 8 \mathrm{~h}$ live time, and with average energy thresholds between $160 \mathrm{GeV}$ and $610 \mathrm{GeV}$, range from $0.4 \%$ to $5.1 \%$ of the Crab Nebula flux. All the upper limits are the most constraining ever reported for these objects.
\end{abstract}

Key words. galaxies: active - BL Lacertae objects: general - gamma rays: observations

\section{Introduction}

Active galactic nuclei are known to emit radiation over the entire electromagnetic spectrum, from radio waves to $\mathrm{TeV}$ $\gamma$-rays. These objects, which are found in only a small fraction of the total number of observed galaxies, are very luminous,

^ UMR 7164 (CNRS, Université Paris VII, CEA, Observatoire de Paris). extremely compact, and can exhibit large luminosity variations on time scales ranging from less than an hour up to several years. Although AGN differ widely in their observed characteristics, a unified description (as reviewed in Urry \& Padovani 1995) has emerged in which an AGN consists of a super-massive black hole $\left(10^{7}-10^{10}\right.$ solar masses $)$ surrounded in the inner regions by an accretion disk, and in the outer regions by a thick torus of gas and dust. In some 
Table 1. Known VHE AGN in order of redshift, along with the references for the initial discovery by other VHE instruments and references for HESS results. Known is defined here as having VHE detections reported by at least two different instruments or by HESS.

\begin{tabular}{cccc}
\hline \hline AGN & $z$ & Reference & HESS Reference \\
\hline M 87 & 0.004 & Aharonian et al. (2003) & Beilicke et al. (2005) \\
Markarian 421 & 0.030 & Punch et al. (1992) & Aharonian et al. (2005c) \\
Markarian 501 & 0.034 & Quinn et al. (1996) & \\
1ES 2344+514 & 0.044 & Catanese et al. (1998) & \\
1ES 1959+650 & 0.047 & Nishiyama et al. (1999) & Aharonian et al. (2005b) \\
PKS 2005-489 & 0.071 & Aharonian et al. (2005a) \\
PKS 2155-304 & 0.116 & Chadwick et al. (1999a) & Horan et al. (2002) \\
1ES 1426+428 & 0.129 & & \\
\hline
\end{tabular}

AGN (the radio-loud population, $\sim 10 \%$ ), a highly relativistic outflow of energetic particles exists approximately perpendicular to the accretion disk and torus plane. This flow forms collimated radio-emitting jets which generate the non-thermal emission observed from radio to $\gamma$-rays. It is believed that some of the numerous AGN classifications result from viewing these objects at various orientation angles with respect to the torus plane. Essentially all AGN detected at VHE energies (shown in order of redshift with references in Table 1) are radio-loud objects of the BL Lacertae (BL Lac) type, which have their jet pointed close to the observer's line of sight. An exception to this exists with the detection of the Fanaroff-Riley type I radio galaxy M 87 at VHE energies. Although M 87 is believed to be a mis-aligned BL Lac (Tsvetanov et al. 1998), it is not clear whether the VHE emission comes from the jet or the central object.

The known VHE AGN have helped to constrain significantly the models for production of VHE $\gamma$-rays through spectral and variability studies. However, there are still many differing models that describe the present data, making a larger sample of known VHE AGN necessary to make more definitive conclusions. Also, VHE photons are absorbed by interactions on the extragalactic background light (EBL) leading to an energy dependent horizon for viewing VHE sources. The energy spectrum of VHE AGN may exhibit characteristics, such as steepening of the spectrum and a cutoff, as a result of this absorption. Interpretation of such features can be used as a probe of the EBL in the optical and near-IR regimes (Stecker et al. 1992; Schroedter 2005), for which direct measurements are dominated by large systematic uncertainties. Since such an interpretation is complicated by discerning which features are a result of EBL absorption and which are intrinsic to the object, a large data set of VHE AGN at differing redshifts are needed to ascertain which effects can be attributed to the EBL.

A large sample of AGN located at $z<0.333$ was observed by HESS in 2003 and 2004. Most of these objects are BL Lacs, many of which are suggested as good candidates for detection as VHE emitters (Costamante \& Ghisellini 2002; Perlman 1999; Stecker et al. 1996). A sample of nearby nonblazar AGN, like M 87, was also observed with the hope of extending the known VHE-bright AGN to other classes. These include a set of famous radio-loud galaxies, characterized by resolved radio, optical and X-ray jets (Cen A, Pictor A, 3C 120, and the quasar 3C 273) and a sample of radio-weak objects (the Seyfert galaxies NGC 1068, NGC 3783 and NGC 7469). The detections resulting from the HESS AGN observation program have been reported elsewhere (see Table 1, for references). These include the confirmation of the VHE emission seen from M 87 and PKS 2155-304, the detection of Markarian 421 using large-zenith-angle observations, and the discovery of VHE emission from PKS 2005-489. Flux upper limits, the strongest ever produced, from the non-detection of the remaining objects are presented here.

\section{HESS detector}

The HESS experiment, a square array (120 m side) of four imaging atmospheric-Cherenkov telescopes located in the Khomas Highland of Namibia $\left(23^{\circ} 16^{\prime} 18^{\prime \prime} \mathrm{S}, 16^{\circ} 30^{\prime} 1^{\prime \prime} \mathrm{E}\right.$, $1800 \mathrm{~m}$ above sea level), uses stereoscopic observations of $\gamma$-ray induced air showers to search for astrophysical $\gamma$-ray emission above $\sim 100 \mathrm{GeV}$. Each telescope has a $107 \mathrm{~m}^{2}$ tessellated mirror dish and a $5^{\circ}$ field-of-view (f.o.v.) camera consisting of 960 individual photomultiplier pixels. The sensitivity of HESS $(5 \sigma$ in $25 \mathrm{~h}$ for a $1 \%$ Crab Nebula flux source at $20^{\circ}$ zenith angle) allows for detection of VHE emission from objects at previously undetectable flux levels. More details on HESS can be found in Bernlöhr et al. (2003), Funk et al. (2004), Hofmann (2003), and Vincent et al. (2003).

\section{Observations}

The HESS observations of AGN in 2004 use the full fourtelescope array. For some of the data, individual telescopes were excluded from the observations or analysis due to hardware problems. Also, 2003 observations of $1 \mathrm{ES} 0323+022$ were made prior to the completion of the array and thus use only two or three telescopes. While the sensitivity of HESS is less during observations with fewer telescopes, it is still unprecedented. Table 2 shows the candidate AGN observed by HESS and gives details of the observations that pass selection criteria which remove data for which the weather conditions were poor or the hardware was not functioning properly. The data were taken in 28 minute runs using Wobble mode, i.e. the source direction is offset, typically by $\pm 0.5^{\circ}$, relative to the center of the f.o.v. of the camera during observations, 


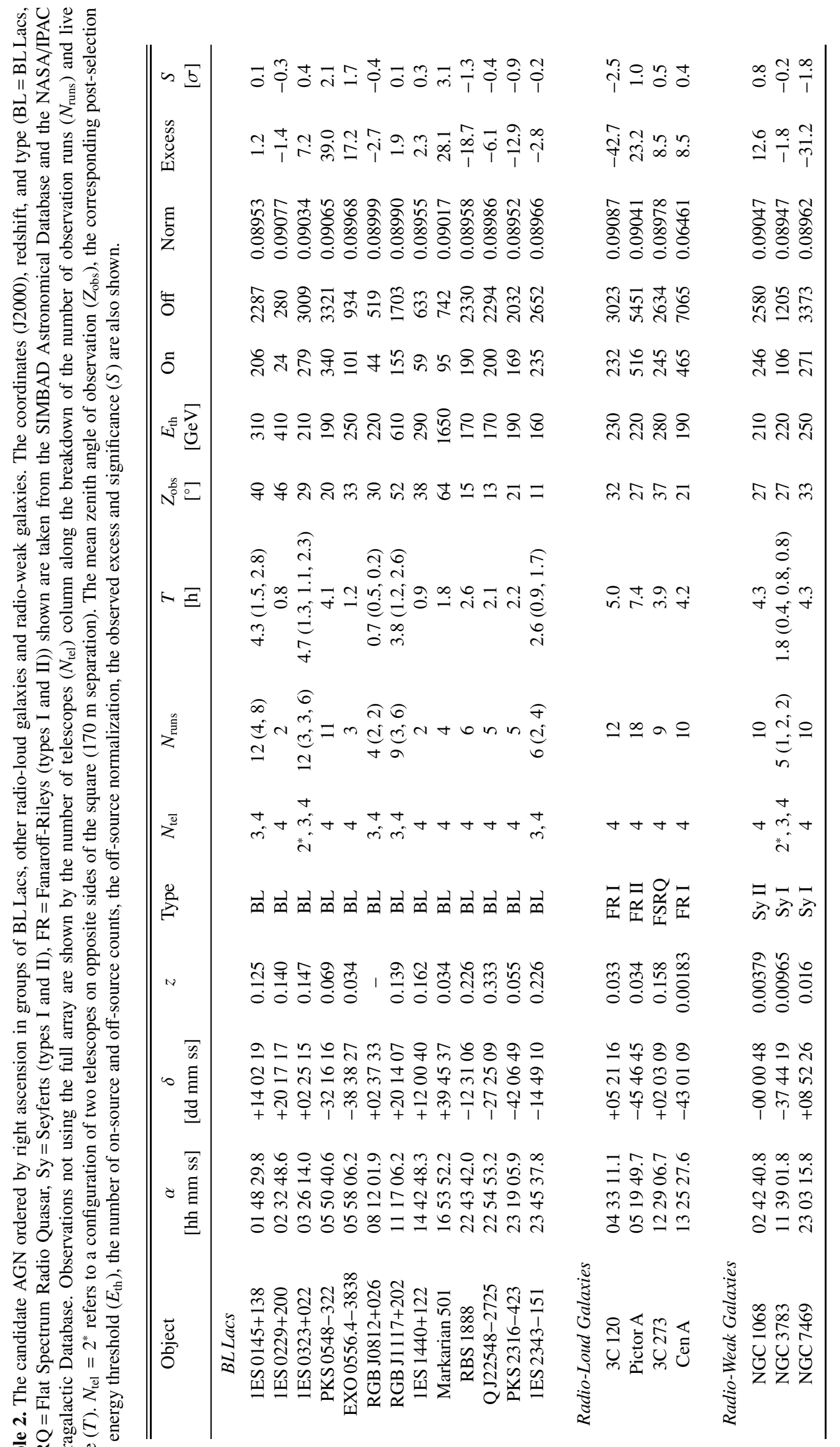


which allows for both on-source observations and simultaneous estimation of the background induced by charged cosmic rays. As the energy threshold of HESS observations increases with zenith angle, the mean zenith angle of the exposure for each of the AGN along with the corresponding average energy threshold (after selection cuts) of those observations is also shown in Table 2. It should be noted that the HESS Monte Carlo simulations show that the azimuthal angle at which an object is observed has a small effect on the energy threshold of observations. Sources which culminate in the south (i.e. those with declination less than the latitude of HESS) have slightly higher energy thresholds (e.g. compare 1ES 0323+022 with Pictor A).

\section{Analysis technique}

The data passing the run selection criteria are calibrated as detailed in Aharonian et al. (2004b), and the event reconstruction and background rejection are performed as described in Aharonian et al. (2005a), with some minor improvements discussed in Aharonian et al. (2005b). The background is estimated using all events passing selection cuts in a number of circular off-source regions offset by the same distance, relative to the center of the f.o.v., in the sky as the on-source region (for more details see Aharonian et al. 2001). The onsource region, the size of which is optimized for the detection of point sources, is a circle of radius $\sim 0.11^{\circ}$ centered on the source, and each off-source region has approximately ${ }^{1}$ the same area as the on-source region. The maximum number of nonoverlapping off-source regions fitting in the field of view are used. An area around the on-source position, completely containing the HESS point-spread-function, is excluded to eliminate possible contamination from poorly reconstructed $\gamma$-rays. For the typical on-source offset of $0.5^{\circ}$, eleven off-source regions are possible. In the case of observations of Cen A, offset by $0.7^{\circ}$, sixteen regions are used. The statistical error on the background measurement is reduced by the use of a larger background sample, and there is no need for a radial acceptance correction, which accounts for the strongest acceptance change across the f.o.v., since the off-source regions are offset by the same radial distance as the on-source region. The significance of any excess is calculated following the method of Eq. (17) in Li \& Ma (1983) and all upper limits are determined using the method of Feldman \& Cousins (1998).

\section{Results}

Figure 1 shows the distribution of the significance observed from the direction of each of the twenty AGN. No significant excess of VHE $\gamma$-rays is found from any of the AGN in the given exposure time $(<8 \mathrm{~h}$ each), with the possible exception of Markarian $501(3.1 \sigma)$. Specific details of the results for each AGN are shown in Table 2. Additionally, a search for serendipitous source discoveries in the HESS f.o.v. centered on each of the AGN yielded no significant excess.

\footnotetext{
${ }^{1}$ The off-source data are first placed into a pixelated twodimensional map and then integrated in an approximate circle for each region. The difference in total area is of order $1 \%$.
}

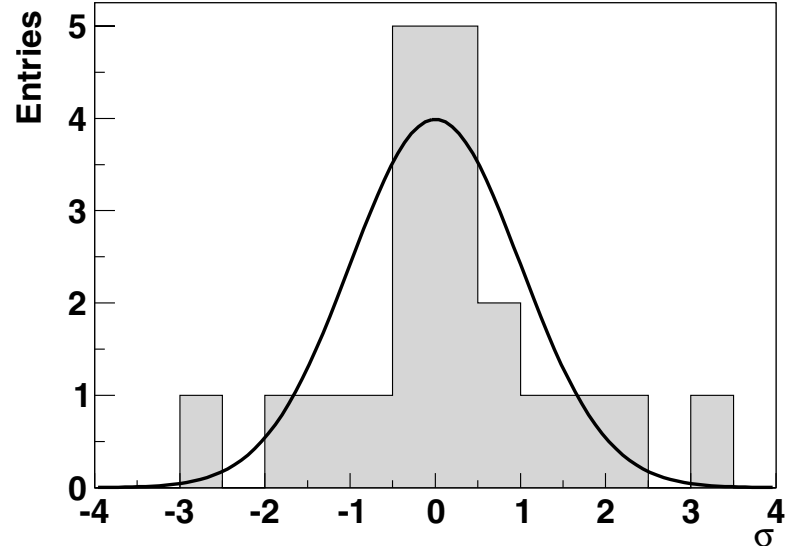

Fig. 1. Distribution of the significance observed from the 20 selected AGN. The curve represents a Gaussian distribution with zero mean and a standard deviation of one.

Given that it is well established that Markarian 501 is a VHE $\gamma$-ray emitter, the excess $(3.1 \sigma)$ from the only night (MJD 53 172) of observations of Markarian 501 can be treated as significant and a flux calculated. Assuming the spectrum measured above $1.5 \mathrm{TeV}$ by the High Energy Gamma Ray Astronomy (HEGRA) experiment (Bradbury et al. 1997), a power law with a photon index of $\Gamma=2.6$, the corresponding integral flux above the $1.65 \mathrm{TeV}$ energy threshold is $I(>1.65 \mathrm{TeV})=\left(1.5 \pm 0.6_{\text {stat }} \pm 0.3_{\text {syst }}\right) \times 10^{-12} \mathrm{~cm}^{-2} \mathrm{~s}^{-1}$ or $\sim 15 \%$ of the HESS Crab Nebula flux above this threshold. While the VHE flux from Markarian 501 is known to be highly variable, the measured flux is similar to the value reported in Bradbury et al. (1997).

For the remaining undetected AGN, 99.9\% upper limits on the integral flux (assuming a power law spectrum with $\Gamma=3.0$ ) above the energy threshold of the observations, and references to previously published limits (when available), are shown in Table 3. The photon index, $\Gamma=3.0$, was chosen for two reasons: First, the recently measured VHE spectra of several AGN (e.g. PKS 2155-304, PKS 2005-489) are softer than the Crab Nebula-like index of $\Gamma=2.5$ often used for VHE upper limits in past publications. Second, the softer index was chosen to account for the possible steepening of the observed spectra of the AGN due to the absorption of $\gamma$-rays on the EBL. Assuming a different photon index (i.e. $\Gamma$ between 2.5 and 3.5) has less than a $\sim 10 \%$ effect on the reported limits, and the systematic error on the upper limits is estimated to be $\sim 20 \%$. The percentage of the Crab Nebula flux shown in Table 3 is calculated relative to the integral flux, above the same threshold, determined from the HESS Crab Nebula spectrum. The HESS limits are considerably ( $>5$ times) stronger than any reported to date. However, due to the generally variable nature of AGN emission, these upper limits constrain the maximum average brightness of the AGN only during the observation time. Hence they are limits on the steady-component or quiescent flux from the AGN. Future flaring behavior may increase the VHE flux from any of these AGN to significantly higher levels.

A search for VHE flux variability from each observed AGN was also performed. Here the nightly integral flux above the average energy threshold was calculated assuming a photon index 
Table 3. Integral flux upper limits $(99.9 \%$ confidence level, assuming a power law spectrum with $\Gamma=3.0)$ above the energy threshold of observations $\left(E_{\mathrm{th}}\right)$ and the corresponding percentage of the HESS Crab Nebula flux from observations of each of the candidate AGN by HESS. References to upper limits available from other VHE instruments are also shown: HEGRA (Aharonian et al. 2004a), Whipple ( $\left({ }^{a}\right.$ : Buckley $1999 ;{ }^{b}$ : de la Calle Perez et al. 2003; ${ }^{c}$ : Horan et al. 2004), CANGAROO $\left({ }^{a}\right.$ : Roberts et al. 1998; ${ }^{b}$ : Roberts et al. 1999; ${ }^{c}$ : Rowell et al. 1999, ${ }^{d}$ : Nishijima 2002), University of Durham Mark VI Telescope ( ${ }^{a}$ : Chadwick et al. 1999b; ${ }^{b}$ : Chadwick et al. 2000), and Milagro (Williams 2004).

\begin{tabular}{|c|c|c|c|c|c|}
\hline Object & $z$ & $\begin{array}{c}E_{\text {th }} \\
{[\mathrm{GeV}]}\end{array}$ & $\begin{array}{c}I\left(>E_{\mathrm{th}}\right) \\
{\left[10^{-12} \mathrm{~cm}^{-2} \mathrm{~s}^{-1}\right]}\end{array}$ & $\begin{array}{c}\text { Crab } \\
\%\end{array}$ & Previous observations \\
\hline \multicolumn{6}{|l|}{ BLLacs } \\
\hline 1ES $0145+138$ & 0.125 & 310 & 2.11 & 1.5 & HEGRA, Whipple ${ }^{c}$ \\
\hline 1ES $0229+200$ & 0.140 & 410 & 2.76 & 3.1 & HEGRA, Whipple ${ }^{b, c}$, Milagro \\
\hline $1 \mathrm{ES} 0323+022$ & 0.147 & 210 & 3.92 & 1.5 & HEGRA, Whipple ${ }^{c}$, Mark VI ${ }^{a}$, Milagro \\
\hline PKS 0548-322 & 0.069 & 190 & 6.65 & 2.2 & Whipple $^{a}$, CANGAROO $^{a, b, d}$, Mark VI $^{b}$ \\
\hline EXO 0556.4-3838 & 0.034 & 250 & 10.1 & 5.1 & \\
\hline RGB J0812+026 & - & 220 & 7.49 & 3.1 & Milagro \\
\hline RGB J1117+202 & 0.139 & 610 & 1.44 & 3.0 & HEGRA, Whipple ${ }^{b}$, Milagro \\
\hline 1ES $1440+122$ & 0.162 & 290 & 5.11 & 3.3 & HEGRA, Milagro \\
\hline RBS 1888 & 0.226 & 170 & 3.19 & 0.9 & \\
\hline Q J22548-2725 & 0.333 & 170 & 5.83 & 1.6 & \\
\hline PKS 2316-423 & 0.055 & 190 & 4.13 & 1.4 & Whipple $^{a}$, CANGAROO $^{a}$, Mark VI $^{a}$ \\
\hline 1ES 2343-151 & 0.226 & 160 & 6.43 & 1.6 & \\
\hline \multicolumn{6}{|l|}{ Radio-Loud Galaxies } \\
\hline $3 \mathrm{C} 120$ & 0.033 & 230 & 0.92 & 0.4 & HEGRA \\
\hline Pictor A & 0.034 & 220 & 3.33 & 1.4 & \\
\hline $3 \mathrm{C} 273$ & 0.158 & 280 & 2.90 & 1.8 & HEGRA, Whipple ${ }^{a}$ \\
\hline Cen A & 0.00183 & 190 & 5.68 & 1.9 & $\mathrm{CANGAROO}^{c}$, Mark VI ${ }^{a}$ \\
\hline \multicolumn{6}{|l|}{ Radio-Weak Galaxies } \\
\hline NGC 1068 & 0.00379 & 210 & 3.28 & 1.3 & \\
\hline NGC 3783 & 0.00965 & 220 & 6.04 & 2.5 & \\
\hline NGC 7469 & 0.016 & 250 & 1.27 & 0.6 & \\
\hline
\end{tabular}

of $\Gamma=3.0$ and fit by a constant. Any flaring behavior would be demonstrated in the form of a poor $\chi^{2}$ probability for the fit. Table 4 shows the dates each AGN was observed and the resulting $\chi^{2}$ probability. As can be seen, no evidence for VHE flux variability is found.

The lack of any significant VHE detection or flaring behavior is perhaps expected from the beahvior of the individual AGN in the X-ray regime. Quick-look results provided by ASM/RXTE team show that none of the AGN (for which allsky monitor data exists) were particularly active during the dates of the HESS observations. On these dates, the measured daily average count rate from each AGN never deviated by more than $\sim 2 \sigma$ from the mean value averaged over the whole $\mathrm{X}$-ray data set.

\section{Discussion}

Since AGN are known to emit radiation in all wavebands, understanding and modelling their emission must take into account their entire spectral energy distribution (SED). Constraining any model is difficult as only a limited number of high-energy measurements currently exist (see Fichtel et al. 1994 for EGRET upper limits on blazars, Seyfert galaxies and radio-loud galaxies). This is especially true at
VHE energies, making the upper limits presented in Table 3 quite useful due to their unprecedented strength. While such modelling is beyond the scope of this paper, the applicability and usefulness of the limits for each of the three classes of observed AGN are discussed.

\subsection{BL lacs}

BL Lacs belong to the sub-class of radio-loud AGN known as blazars, which are AGN thought to possess a jet which is viewed close to the line of sight (Urry \& Padovani 1995). The distinction between BL Lacs and other blazars is primarily based on their optical spectra which are characterized by weak or absent emission lines. As mentioned in the introduction, almost all VHE bright AGN belong to this class. These AGN have dominantly non-thermal emission and are characterized by a double-humped SED. The low-energy component is widely accepted as originating from synchrotron radiation of relativistic electrons in the magnetic field around the object. However, the origin of the high-energy component is the subject of much debate. Various models involving either leptonic or hadronic processes have been proposed and can be constrained using the HESS results. However, some caveats are required for interpreting a blazar SED with the HESS upper limits. 
Table 4. The dates of the HESS observations of each AGN and the $\chi^{2}$ probability for a fit of a constant to the nightly integral flux.

\begin{tabular}{|c|c|c|}
\hline Object & MJD-50000 & $\mathrm{P}\left(\chi^{2}\right)$ \\
\hline \multicolumn{3}{|l|}{ BLLacs } \\
\hline 1ES $0145+138$ & $3202,3205,3210-14$ & 0.85 \\
\hline $1 \mathrm{ES} 0229+200$ & 3317 & - \\
\hline $1 \mathrm{ES} 0323+022$ & $2904-05,3267$ & 0.87 \\
\hline PKS 0548-322 & $3296,3299-300,3303$ & 0.83 \\
\hline EXO 0556.4-3838 & 3347,3354 & 0.67 \\
\hline RGB J0812+026 & 3081 & - \\
\hline RGB J1117+202 & $3054,3112,3114,3116$ & 0.85 \\
\hline 1ES $1440+122$ & 3110,3119 & 0.13 \\
\hline RBS 1888 & $3207-08,3210$ & 0.72 \\
\hline Q J22548-2725 & $3201,3210-11$ & 0.90 \\
\hline PKS 2316-423 & $3201,3207-08$ & 0.50 \\
\hline 1ES $2343-151$ & $3211-13$ & 0.71 \\
\hline \multicolumn{3}{|l|}{ Radio-Loud Galaxies } \\
\hline $3 \mathrm{C} 120$ & $3316-19,3353-55$ & 0.81 \\
\hline Pictor A & $3269,3318-19,3351,3353-54$ & 0.35 \\
\hline $3 \mathrm{C} 273$ & $3109-10,3148-49$ & 0.25 \\
\hline Cen A & $3111-13$ & 0.13 \\
\hline \multicolumn{3}{|l|}{ Radio-Weak Galaxies } \\
\hline NGC 1068 & 3290, 3292-94, 3296 & 0.55 \\
\hline NGC 3783 & $3107-08$ & 0.52 \\
\hline NGC 7469 & $3202,3206,3211-12$ & 0.89 \\
\hline
\end{tabular}

Blazars are known to be highly variable at all wavelengths, typically characterized by low-emission quiescent states interrupted by periods of flaring behavior where the flux increases dramatically. In some cases this increase is several orders of magnitude. Due to this extreme variability, it has been shown that fitting the SED of blazars has very large uncertainties when non-simultaneous multiwavelength (MWL) data are used (see e.g. Böttcher et al. 2002). As a result the usefulness of nonsimultaneous upper limits, as is the case for the HESS observations, in modelling these object is limited. The HESS upper limits, in the absence of simultaneous MWL data, are only relevant for modelling the quiescent state of the blazar using archival low-state MWL data. An additional problem using these upper limits arises due to the absorption of VHE photons on the EBL. The upper limit on the flux intrinsic to the object can be significantly higher than those presented in Table 3 depending on the redshift. As a result the upper limits must have the effects of the EBL removed before they can be used for modelling. Unfortunately, parameterizations of the EBL are poorly constrained leading to numerous models with dramatically different behaviors, adding another significant uncertainty when using VHE upper limits to help model blazar emission. Given the wide range of EBL interpretations, this deabsorption is not performed here.

Taking note of the caveats regarding the effects of the EBL and the issues with non-simultaneous observations, a comparison of the upper limits is made, where possible, to three sets of VHE flux predictions based on the SEDs of blazars.
Table 5. Integral flux upper limits ( $99.9 \%$ confidence level, $\Gamma=3.0$ ) scaled to above $300 \mathrm{GeV}$, and above $1 \mathrm{TeV}$, from HESS observations for the selected AGN where any of the SDS, FOS and $C G$ predictions are available. The units are $10^{-12} \mathrm{~cm}^{-2} \mathrm{~s}^{-1}$. The cases for which the HESS upper limit is below the predicted flux are shown in bold.

\begin{tabular}{cccc}
\hline \hline Blazar & $\begin{array}{c}\text { HESS } \\
300 \mathrm{GeV}\end{array}$ & $\begin{array}{c}\text { HESS } \\
1 \mathrm{TeV}\end{array}$ & Pred. \\
\hline $0145+138$ & 2.25 & 0.203 & SDS \\
$0229+200$ & 5.15 & 0.464 & SDS, FOS, $C G$ \\
$0323+022$ & 1.92 & 0.173 & $S D S$, FOS, $C G$ \\
$0548-322$ & 2.67 & 0.240 & SDS, FOS, $C G$ \\
$0556-384$ & 7.03 & 0.632 & FOS \\
$0812+026$ & 4.03 & 0.363 & FOS \\
$1117+202$ & 5.99 & 0.539 & FOS \\
$1440+122$ & 4.77 & 0.429 & $S D S$, FOS,$C G$ \\
\hline
\end{tabular}

The first set (Stecker et al. 1996), referred to as SDS henceforth, uses simple scaling arguments to predict VHE fluxes for Einstein Slew survey objects. In the case of the SDS flux predictions the effects of EBL absorption are already accounted for with an "averaged" model. The other two sets of predictions are taken from Costamante \& Ghisellini (2002). The first, referred to as $F O S$, uses a phenomenological description of the average SED of blazars based on their bolometric luminosity (Fossati et al. 1998), modified by Donato et al. (2001), and derives predictions on the basis of the individual blazar's radio luminosity and synchrotron peak frequency. The second, referred to as $C G$, uses fits of a synchrotron self-Compton model to existing multiwavelength data. Both the FOS and $C G$ predictions do not have the effects of EBL absorption accounted for. This could change the flux predictions by factors of $\sim 5$ above $300 \mathrm{GeV}$ and by factors $>100$ above $1 \mathrm{TeV}$ for objects at $z \sim 0.2$.

Table 5 shows the $99.9 \%$ HESS flux upper limits extrapolated (assuming $\Gamma=3.0$ ) to above $300 \mathrm{GeV}$ and above $1 \mathrm{TeV}$, as well as which predictions are available above these thresholds. The HESS upper limits are below the SDS predictions above $300 \mathrm{GeV}$ in three of the five cases (factors ranging from $\sim 2$ to $\sim 5$ ), and below two of the five predictions above $1 \mathrm{TeV}$ (factors of $\sim 1.3$ and $\sim 5$ ). Even if the EBL absorption effects are accounted for in the $S D S$ predictions, the discrepancies can easily be accounted for by the aforementioned simultaneity caveats and thus the HESS upper limits do not make any strong statements regarding the $S D S$ predictions. All the FOS predictions are above the HESS upper limits, from factors of $\sim 1.4$ to $\sim 16$ for the predictions above $300 \mathrm{GeV}$ and factors of $\sim 5$ to $\sim 40$ for the predictions above $1 \mathrm{TeV}$. While at first this seems severe, accounting for $\mathrm{TeV}$ absorption can reduce these discrepancies dramatically. In addition the FOS predictions are claimed to be more suitable for "high" state VHE flux predictions, whereas the HESS upper limits are most appropriate for constraining the quiescent state of the AGN. Given that variability of up to two orders of magnitude have been seen in VHE blazars such as Markarian 421, it is clear that it is again difficult to test these predictions with the HESS upper limits. However, the disagreement suggests that different sets of parameters might be 
necessary to account for the quiescent state of the source. The $C G$ predictions, which are claimed to be more appropriate for the quiescent state of the AGN tested by the HESS upper limits, are all below the upper limits.

\subsection{Other radio-loud galaxies}

Speculation exists for detectable levels of VHE emission from the jets of AGN without doppler boosting along the line of sight (see e.g. Aharonian et al. 1994). Therefore, the HESS observation program also included four other radio-loud AGN. Like BL Lacs they all possess jets. One of these, 3C 273, meets some, but not all, of the phenomenological criteria for classification as a blazar. However, it is most accurately characterized as a quasar. It is the brightest and one of the most nearby quasars. The other three AGN, Cen A, 3C 120 and Pictor A, are found in Fanaroff-Riley (FR) radio galaxies. These galaxies fall into two classes, FR I and FR II. The distinction is based on their radio morphology (Fanaroff \& Riley 1974). FR I objects, such as Cen A (the prototype), 3C 120 and the VHE-emitter M 87, show extended jets with no distinct termination point, and FR II objects, like Pictor A, have narrow, collimated jets with terminal "hotspots". These FR objects differ from BL Lacs mainly due to a large viewing angle $\left(50^{\circ}-80^{\circ}\right)$ with respect to the jet axis.

Chandra observations (for a review see e.g. Harris 2001) show that Pictor A, 3C 120, and 3C 273 all possess bright X-ray features like knots and hot spots in their large-scale extragalactic jets. The X-ray fluxes of these features are at least a factor of 10 larger than the radio and optical fluxes. This behavior is the opposite of the predictions from synchrotron self-Compton and inverse-Compton models and requires alternative theoretical explanations (see e.g. Aharonian 2002). Use of the HESS upper limits for these objects should aid in constraining some of the presented scenarios. However, they are still subject to the aforementioned variability and EBL absorption (mainly for 3C 273) caveats.

Located at a distance of $3.4 \mathrm{Mpc}$, Cen A (NGC 5128) is the closest radio-loud AGN. It is one of the best-studied extragalactic objects due to its large apparent brightness in all wavebands (for a recent review see Israel 1998). The proximity of Cen A means that the intrinsic spectrum of the object is unaffected by absorption on the EBL, considerably simplifying the use of the HESS upper limit in the modelling of its VHE emission. However, the lack of simultaneous observations is still an issue as Cen A, like blazars, exhibits large flux variability, albeit on much longer time scales (years).

During the early days of VHE astronomy, a detection of emission above $300 \mathrm{GeV}$ from Cen A was claimed using a non-imaging Cherenkov system (Grindlay et al. 1975) during a historically high emission state. The flux reported, $I(>300 \mathrm{GeV})=(4.4 \pm 1.0) \times 10^{-11} \mathrm{~cm}^{-2} \mathrm{~s}^{-1}$, is over an order of magnitude above the HESS $99.9 \%$ flux upper limit extrapolated to above $300 \mathrm{GeV}, I(>300 \mathrm{GeV})<2.3 \times 10^{-12} \mathrm{~cm}^{-2} \mathrm{~s}^{-1}$. The HESS result does not contradict the claimed detection as RXTE ASM observations show that Cen A was in a low emission state when observed by HESS. During a similar low emission state, EGRET detected $>100 \mathrm{MeV} \gamma$-ray emission from Cen A (Sreekumar et al. 1999). This is the only EGRET detection associated with an AGN that is not a member of the blazar class. Extrapolating the EGRET spectrum to above the HESS threshold yields $I(>190 \mathrm{GeV})=3.5 \times 10^{-12} \mathrm{~cm}^{-2} \mathrm{~s}^{-1}$ which is $\sim 60 \%$ of the upper limit shown in Table 3 . The HESS limit is similar, $5.5 \times 10^{-12} \mathrm{~cm}^{-2} \mathrm{~s}^{-1}$, when assuming the measured EGRET spectrum of $\Gamma=2.40$. These results imply that future identification of a high-emission state in Cen A should motivate further VHE observations.

\subsection{Radio-weak galaxies}

All of the radio-weak AGN observed by HESS are located in Seyfert galaxies which differ from the galaxies previously discussed in many respects. Like the other AGN, they have outflows, albeit typically with low velocity and uncollimated, approximately perpendicular to the accretion disk. Some even have collimated jets that emit synchrotron radiation. However, the jets are neither as collimated as in radioloud AGN, nor do they show any indications of relativistic motion. Two kinds of Seyfert galaxies (types I and II) exist whose differences can be explained in terms of viewing angle (Antonucci \& Miller 1985). It is believed that Seyfert I galaxies are viewed "face on" and thus the nuclear regions are directly visible, whereas Seyfert II galaxies are viewed "edge on" causing the nuclear regions to be obscured by material (the torus or warped disk). Currently, no Seyfert galaxies are known to be VHE emitters.

Three bright well-studied Seyfert galaxies were observed by HESS: NGC 1068, NGC 3783, and NGC 7469. NGC 1068 (M 77), the prototypical type II object, is the brightest and closest known Seyfert galaxy and as such is perhaps the best candidate for detection of this class at VHE energies. Here it should be noted that since the emission from Seyferts is not beamed, their orientation is not as important as with blazars. NGC 3783, a classical type I object, is also one of the brightest and closest Seyfert galaxies, and one of the most well studied. It is also interesting in that exceptionally deep measurements made using the Chandra X-ray Observatory reveal a fast $\left(>10^{6} \mathrm{~km} \mathrm{~h}^{-1}\right)$ wind of highly ionized atoms blowing away from the galaxy's suspected central black hole (Kaspi et al. 2002). NGC 7469, also type $\mathrm{I}$, is unusual in that it has an inner ring of gas very close to the nucleus that is undergoing massive star formation (Genzel et al. 1995).

None of these objects were detected and the upper limits shown in Table 3 are quite constraining. While Seyfert-type galaxies are not necessarily expected to emit VHE $\gamma$-rays at observable levels, the HESS results easily provide constraints for modelling. This is because these AGN generally show less variability than blazars, and all the ones observed are close enough to only have minimal effects from the absorption of VHE photons on the EBL. The HESS results could be interpreted as implying that Seyfert-type AGN are not significant emitters of VHE photons. However, the observed sample and exposure times are small, making it premature to rule the class out all together. 


\section{Conclusions}

HESS observed greater than twenty AGN in 2003 and 2004 as part of a campaign to identify new VHE-bright AGN. Several significant detections from this campaign have been reported elsewhere (see Table 1, for references). Results presented here detail the AGN observations for which no significant excess was found, apart from a marginal signal from the well-known VHE-emitter Markarian 501. Despite the limited exposure $(<8 \mathrm{~h})$ for each of these AGN, the upper limits on the VHE flux determined by HESS are the most stringent to date, demonstrating the unprecedented sensitivity of the instrument. Clearly the strength of the limits makes them quite useful, yet it must again be stressed that any interpretation using the HESS limits must take into account both the EBL and the state of the source using simultaneous data at different wavelengths.

The HESS AGN observation program is not complete as many proposed candidates have not yet been observed. Further, more time is scheduled for observations of some of the AGN presented here as part of a monitoring effort for blazars. HESS has already detected $\gamma$-ray emission from four AGN, including one (PKS 2005-489) never previously detected in the VHE regime. Clearly the prospects of finding additional VHE-bright AGN are excellent.

Acknowledgements. The support of the Namibian authorities and of the University of Namibia in facilitating the construction and operation of HESS is gratefully acknowledged, as is the support by the German Ministry for Education and Research (BMBF), the MaxPlanck-Society, the French Ministry for Research, the CNRS-IN2P3 and the Astroparticle Interdisciplinary Programme of the CNRS, the UK Particle Physics and Astronomy Research Council (PPARC), the IPNP of the Charles University, the South African Department of Science and Technology and National Research Foundation, and by the University of Namibia. We appreciate the excellent work of the technical support staff in Berlin, Durham, Hamburg, Heidelberg, Palaiseau, Paris, Saclay, and in Namibia in the construction and operation of the equipment.

\section{References}

Aharonian, F. A., Coppi, P. S., \& Völk, H. J. 1994, ApJ, 423, L5

Aharonian, F., Akhperjanian, A., Barrio, J., et al. 2001, A\&A, 370, 112

Aharonian, F. 2002, MNRAS, 332, 215

Aharonian, F., Akhperjanian, A., Beilicke, M., et al. 2003, A\&A, 403, L1

Aharonian, F., Akhperjanian, A., Beilicke, M., et al. 2004a, A\&A, 421,529

Aharonian, F., Akhperjanian, A. G., Aye, K.-M., et al. 2004b, Astropart Phys., 22, 109

Aharonian, F., Akhperjanian, A. G., Aye, K.-M., et al. 2005a, A\&A, 430,865

Aharonian, F., Akhperjanian, A. G., Aye, K.-M., et al. 2005b, A\&A, 436, L17

Aharonian, F., Akhperjanian, A. G., Aye, K.-M., et al. 2005c, A\&A, 437,95
Antonucci, R. R., \& Miller, J. S. 1985, ApJ, 297, 621

Beilicke, M., Cornils, R., Heinzelmann, G., et al. 2005, Proc. 22nd Texas Symp. Relativistic Astrophys. (Stanford)

Bernlöhr, K., Carrol, O., Cornils, R., et al. 2003, Astropart Phys., 20, 111

Böttcher, M., Mukherjee, R., \& Reimer, A. 2002, ApJ, 581, 143

Bradbury, S. M., Deckers, T., Petry, D., et al. 1997, A\&A, 320, L5

Buckley, J. 1999, Astropart. Phys., 11, 119

Catanese, M., Akerlof, C. W., Badran, H. M., et al. 1998, ApJ, 501, 616

Chadwick, P. M., Lyons, K., McComb, T. J. L., et al. 1999a, ApJ, 513, 161

Chadwick, P. M., Lyons, K., McComb, T. J. L., et al. 1999b, ApJ, 521, 547

Chadwick, P. M., Daniel, M. K., Lyons, K., et al. 2000, A\&A, 364, 450

Costamante, L., \& Ghisellini, G. 2002, A\&A, 384, 56

de la Calle Perez, I., Bond, I. H., Boyle, P. J., et al. 2003, ApJ, 599, 909

Donato, D., Ghisellini, G., Tagliaferri, G., et al. 2001, A\&A, 375, 739

Fanaroff, B. L., \& Riley, J. M. 1974, MNRAS, 167, 31

Feldman, G. J., \& Cousins, R. D. 1998, Phys. Rev. D, 57, 3873

Fichtel, C. G., Bertsch, D. L., Chiang, J., et al. 1994, ApJS, 94, 551

Fossati, G., Maraschi, L., Celotti, A., et al. 1998, MNRAS, 299, 433

Funk, S., Hermann, G., Hinton, J., et al. 2004, Astropart. Phys., 22, 285

Genzel, R., Weitzel, L., Tacconi-Garman, L. E., et al. 1995, ApJ, 444, 129

Grindlay, J. E., Helmken, H. F., Brown, R. H., et al. 1975, ApJ, 197, L9

Harris, D. E. 2001, ASP Conf. Proc., 250, 204

Hofmann, W. 2003, Proc. 28th ICRC (Tsukuba), 2811

Horan, D., Badran, H. M., Bond, I. H., et al. 2002, ApJ, 571, 753

Horan, D., Badran, H. M., Bond, I. H., et al. 2004, ApJ, 603, 51

Israel, F. P. 1998, A\&AR, 8, 237

Kaspi, S., Brandt, W. N., George, I. M., et al. 2002, ApJ, 574, 643

Li, T., \& Ma, Y. 1983, ApJ, 272, 317

Nishijima, K. 2002, Publ. Astron. Soc. Aust., 19, 26

Nishiyama, T., Chamoto, N., Chikawa, M., et al. 1999, Proc. 26th ICRC (Salt Lake City), 3, 370

Perlman, E. S. 1999, AIP Conf. Proc., 515, 53

Punch, M., Akerlof, C. W., Cawley, M. F., et al. 1992, Nature, 358, 477

Quinn, J., Akerlof, C. W., Biller, S. D., et al. 1996, ApJ, 456, L83

Roberts, M. D., Dazeley, S. A., Edwards, P. G., et al. 1998, A\&A, 337, 25

Roberts, M. D., McGee, P., Dazeley, S. A., et al. 1999, A\&A, 343, 691

Rowell, G. P., Dazeley, S. A., Edwards, P. G., et al. 1999, Astropart. Phys., 11, 217

Schroedter, M. 2005, ApJ, in Press [arXiv: astro-ph/0504397]

Sreekumar, P., Bertsch, D. L., Hartmann, R. C., et al. 1999, Astropart. Phys., 11, 221

Stecker, F. W., de Jager, O. C., \& Salamon, M. H. 1992, ApJ, 390, 49

Stecker, F. W., de Jager, O. C., \& Salamon, M. H. 1996, ApJ, 473, L75

Tsvetanov, Z. I., Hartig, G. F., Ford, H. C., et al. 1998, ApJ, 493, L83

Urry, C. M., \& Padovani, P. 1995, PASP, 107, 803

Vincent, P., Denance, J.-P., Huppert, J.-F., et al. 2003, Proc. 28th ICRC (Tsukuba), 2887

Williams, D. 2004, AIP Conf. Proc., 745, 499 